\title{
Nine-Year Follow-Up of Intacs Implantation for Keratoconus
}

\author{
George D. Kymionis, Michael A. Grentzelos, Vasilios F. Diakonis*, Aristofanis I. Pallikaris and \\ Ioannis G. Pallikaris
}

Institute of Vision and Optics, Department of Ophthalmology, Medical School, University of Crete, Greece

\begin{abstract}
Purpose: To present a case of nine- year bilateral Intacs (Addition Technology, Inc, Fremont, California, USA) implantation for early stage keratoconus.

Methods: A 25-year-old male underwent bilateral Intacs implantation for the management of keratoconus and hardcontact-lens intolerance (stage 1) in 1999.

Results: Nine years postoperatively, spherical equivalent refraction changed from preoperative -0.75 and -2.25 to +0.75 and -1.25 for the right and the left eye, respectively. UCVA was improved from 20/50 to 20/25 in the right and from $20 / 200$ to $20 / 32$ in the left eye. BSCVA of 20/20 in the right eye maintained stable in comparison with the Pre-Intacs BSCVA, while BSCVA was improved from $20 / 25$ to $20 / 20$ in the fellow eye. No early or late complications were observed.

Conclusions: Nine years after bilateral Intacs implantation for the management of early stage keratoconus, there was a significant improvement and postoperative stability in patient's visual acuity. No long-term, sight-threatening complications were identified during follow-up.
\end{abstract}

\section{INTRODUCTION}

Keratoconus is a non-inflammatory, naturally occurring ectatic corneal condition in which the cornea gets thinner and steeper over time [1]. This leads to corneal biomechanical instability, resulting in myopia, irregular astigmatism and loss of best-spectacle corrected visual acuity [2]. The improvement of visual acuity in keratoconic patients is conventionally achieved using either spectacles or contact lenses. In cases where these methods are impossible, minimal invasive techniques, such as intra-corneal ring segment implantation, have been proposed and demonstrated satisfactory results $[3,4]$.

Corneal ring segment implantation was the first nonlaser, and tissue-saving procedure approved by the Food and Drug Administration (FDA) of the United States for the correction of myopia. Its capability to flatten the central part of cornea was also evaluated for keratoconic eyes which demonstrate an abnormal corneal surface, which in turn influences the visual performance of patients. The use of intra-corneal ring segments for the management of keratoconus has demonstrated satisfactory results, since in the majority of the patients improvement in uncorrected (UVA) and best corrected (BSCVA) visual acuity has been documented $[5,6]$.

There are a few studies in the literature with long-term follow-up of Intacs microthin prescription inserts for the management of keratoconus $[5,6]$, but none refers to a nineyear follow-up. In this case report we present a patient of

*Address correspondence to this author at the Institute of Vision \& Optics, Department of Medicine, University of Crete, 71003 Heraklion, Crete, Greece; Tel: ++302810.371800; Fax: ++302810.394653;

E-mail: diakonis@gmail.com nine years after bilateral Intacs implantation for the management of keratoconus.

\section{CASE REPORT}

A 25-year-old male underwent bilateral intrastromal corneal ring segments (Intacs) implantation in both eyes for progressive keratoconus stage 1 [7] (keratoconus stage 1 is defined as: eccentric corneal steepening, induced myopia and/or astigmatism $<5 \mathrm{D}$, Corneal radii $<48 \mathrm{D}$ and Vogt's striae with no scar formation) and hard-contact-lens intolerance in 1999 (the patient was referred to our institute for progressive keratoconus, decrease in visual acuity and corneal topographic changes). Systemic history was unremarkable. Preoperative examination included uncorrected visual acuity (UCVA), best spectacle corrected visual acuity (BSCVA), manifest and cycloplegic refraction, intraocular pressure (IOP), corneal topography (EyeSys Technologies, Houston, Texas, USA), pachymetry of central and peripheral cornea and slit-lamp microscopy. UCVA was $20 / 50$ in the right eye and 20/200 in the left eye. BSCVA was $20 / 20$ with refraction of $0.00-1.50 \times 65$ in the right eye and 20/25 with refraction of $-1.25-2.25 \times 120$ in the fellow eye. Intraocular pressure was $12 \mathrm{mmHg}$ in both eyes. Keratometric values were $44.00 / 43.38 \mathrm{D}$ at the right eye and 45.30/44.29 D at the left eye (Fig. 1). Central corneal thickness was $509 \mu \mathrm{m}$ and $532 \mu \mathrm{m}$ in the right and the left eye, respectively. Ophthalmic examination showed no other anterior or posterior segment abnormalities.

The surgical procedure was conducted under sterile conditions and topical anesthesia with proxymetacaine hydrochloride $0,5 \%$ eyedrops (Alcaine, Alcon Labs). The corneal center was marked by indentation of the epithelium with a Sinskey hook and it was based on the center of the pupillary aperature. The entrance incision was placed at the 


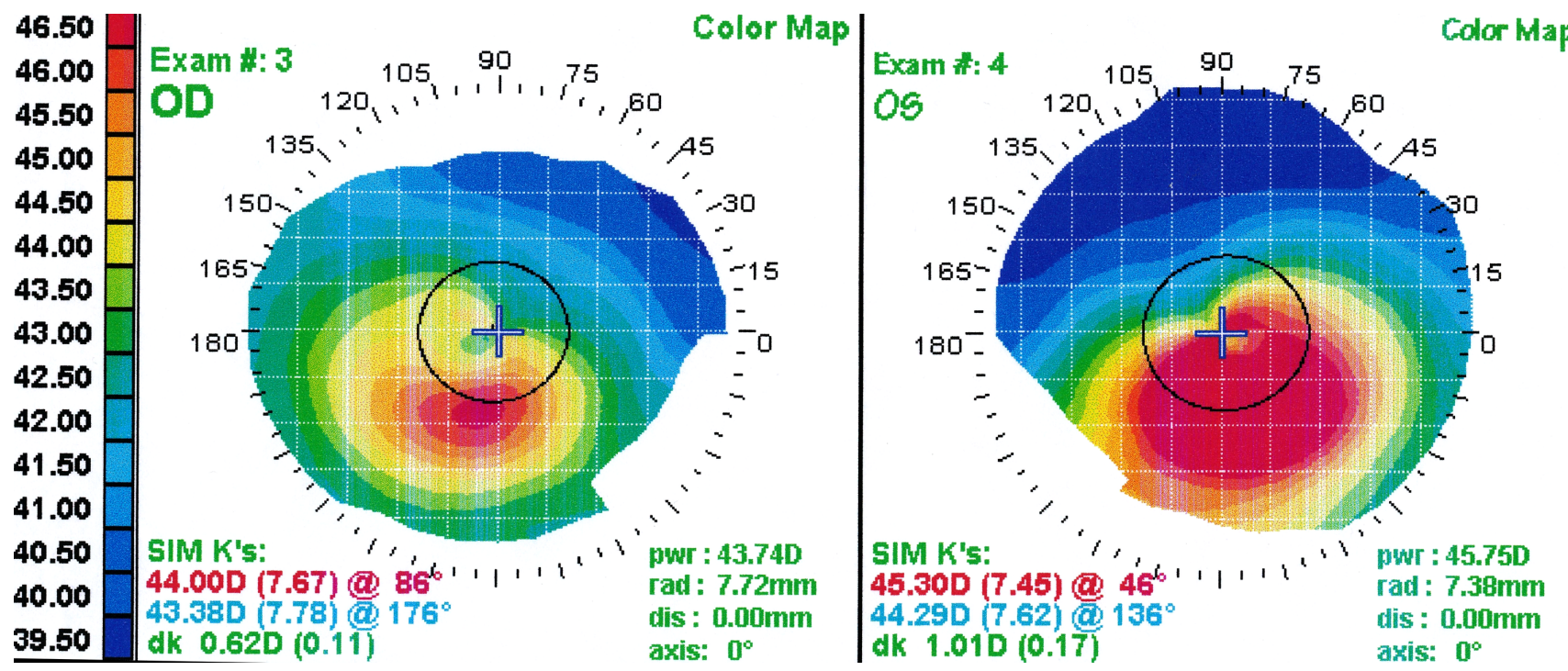

Fig. (1). Preoperative - (left topography, right eye and right topography, left eye) topographic maps.

topographic steep axis. The corneal thickness was measured intraoperatively at the incision site and peripherally in the cornea along the ring placement markings with ultrasonic pachymetry (Sonogage, Cleveland, Ohio, USA). Using a diamond knife, set at $70 \%$ of the thinnest corneal measurement, a $0.9-\mathrm{mm}$ radial incision was formed at $410 \mu \mathrm{m}$ and $420 \mu \mathrm{m}$ of the right and the left eye respectively, and two intrastromal corneal pockets were created using two Sinskey hooks and a Suarez spreader. Two Intacs segments of $0.45-\mathrm{mm}$ thickness were inserted maintaining a space of approximately $2.0 \mathrm{~mm}$ between their ends and $1.5 \mathrm{~mm}$ between the opposite edge of each segment and the edge of the incision. The incision site was sutured using a single 10/0 nylon stitch. The sutures were removed two weeks after surgery.

Three months postoperatively, UCVA was 20/25 in both eyes and BCVA was 20/20 in both eyes with refraction of $+1.00-1.25 \times 30$ in the right eye and $-0.50-1.25 \times 145$ in the left eye. Two years postoperatively, UCVA was 20/25 in the right eye and 20/32 in the fellow eye. BCVA was 20/20 in both eyes with $+0.75-1.50 \times 30$ in the right eye and -1.50 $-1.50 \times 150$ in the fellow eye. Keratometric measurements were $42.85 / 40.85 \mathrm{D}$ in the right eye and 45.54/42.76 D in the left eye (Figs. 2, 3).
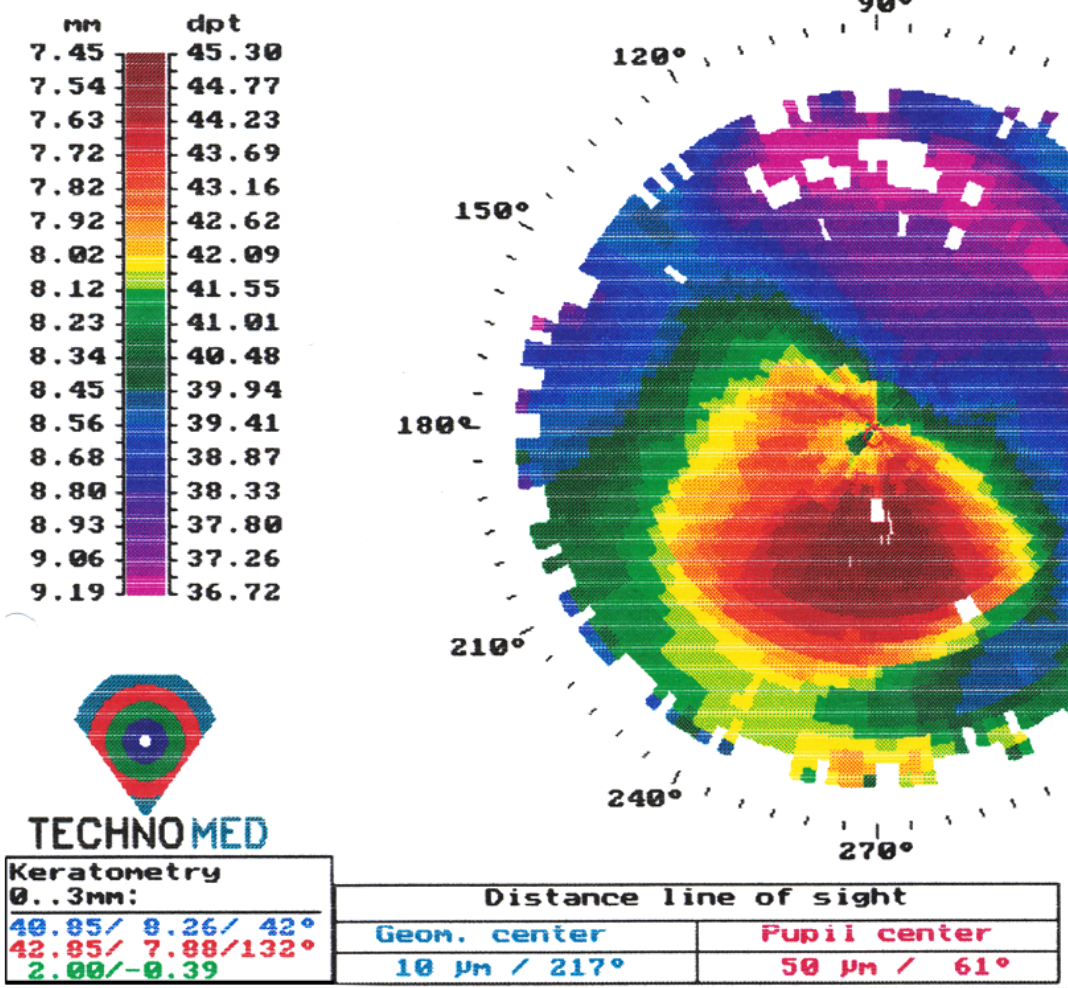

Fig. (2). Two years postoperative topography of the right eye. 

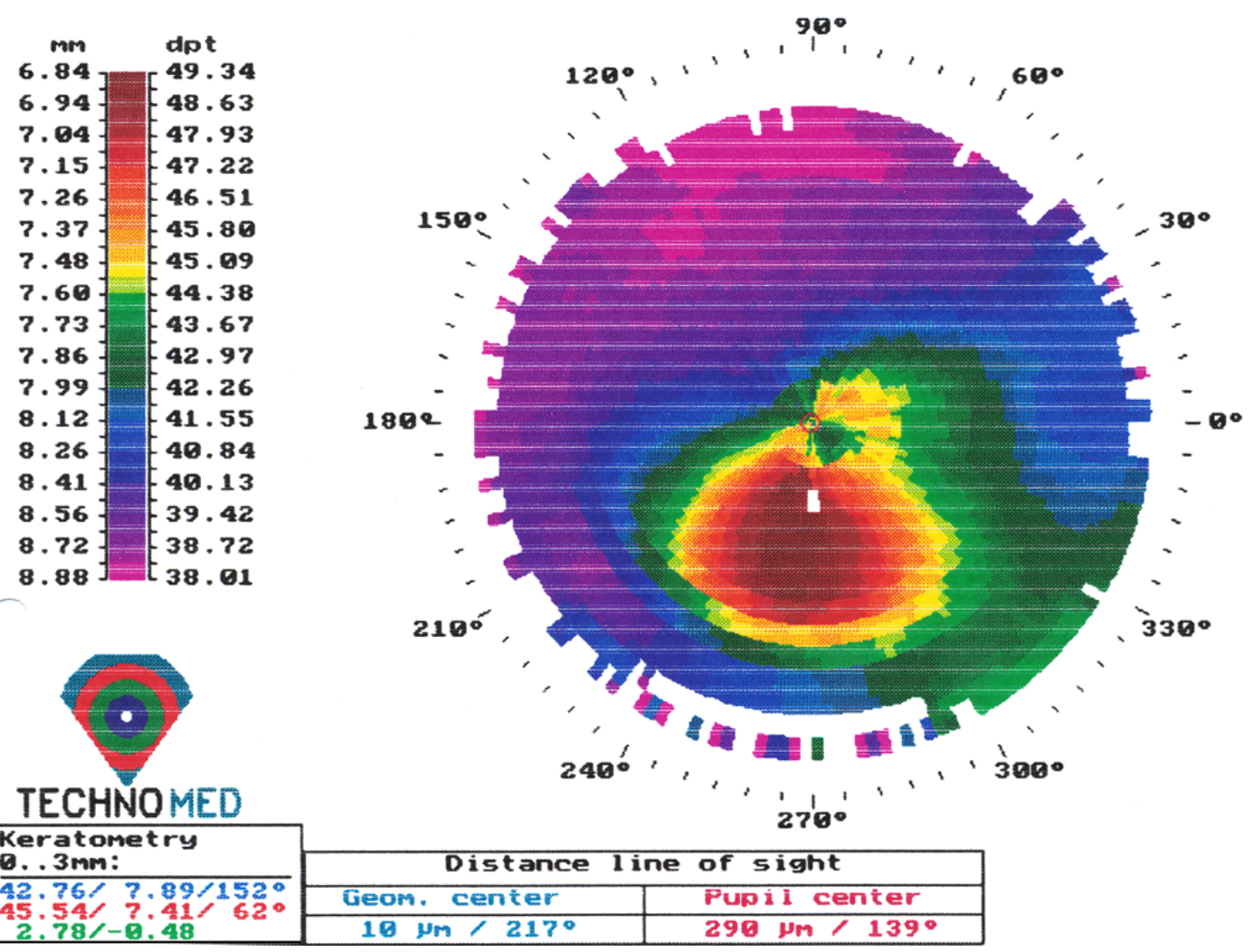

Fig. (3). Two years postoperative topography of the left eye.

Nine years postoperatively, UCVA was 20/25 in the right eye and 20/32 in the left eye. BCVA was 20/20 in both eyes with refraction of $+1.50-1.50 \times 45$ in the right eye and -1.00 $-0.75 \times 115$ in the fellow eye. Topographic improvement and stability was observed (42.86/41.09 in the right eye,
44.54/42.02 in the left eye) nine years postoperatively in comparison with the preoperative and two year follow up topographic findings (Figs. 4, 5). Slit-lamp examination revealed deposits in the lamellar channel around the segments of both eyes (Fig. 6).
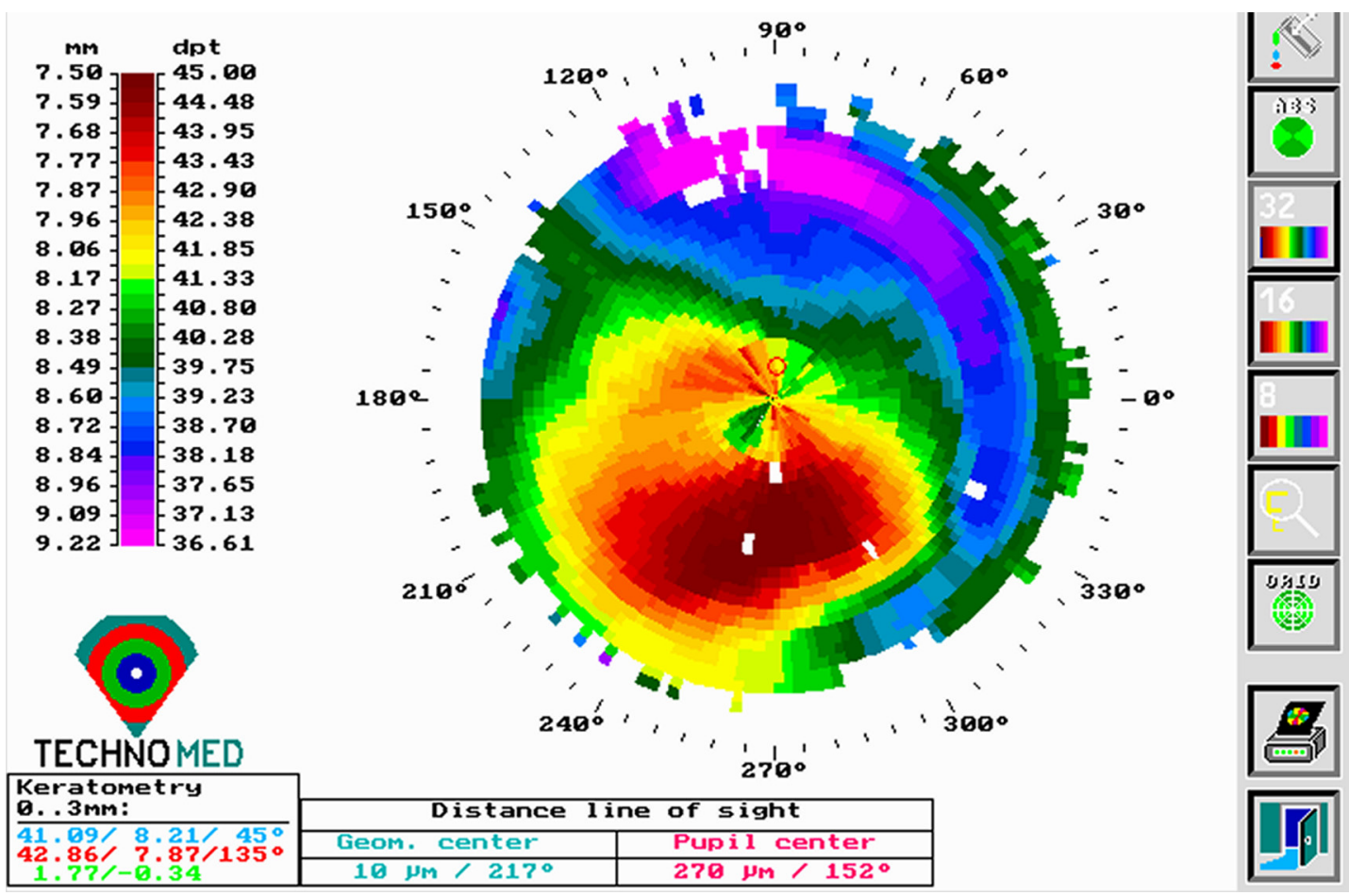

Fig. (4). Nine years postoperative topography of the right eye, postoperative topographies showed a topographic improvement and stability. 

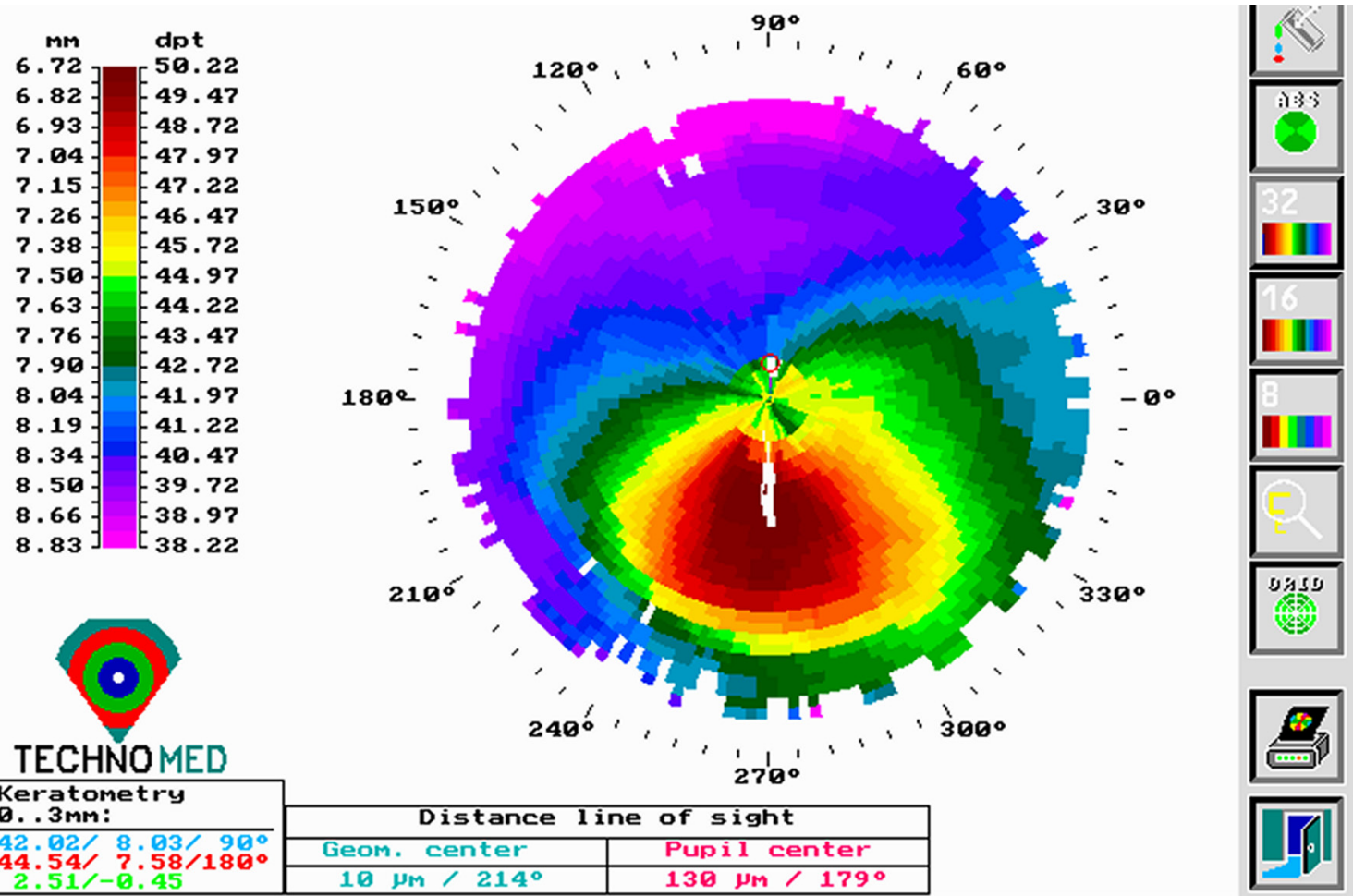

Fig. (5). Nine years postoperative topography of the left eye, postoperative topographies showed a topographic improvement and stability.

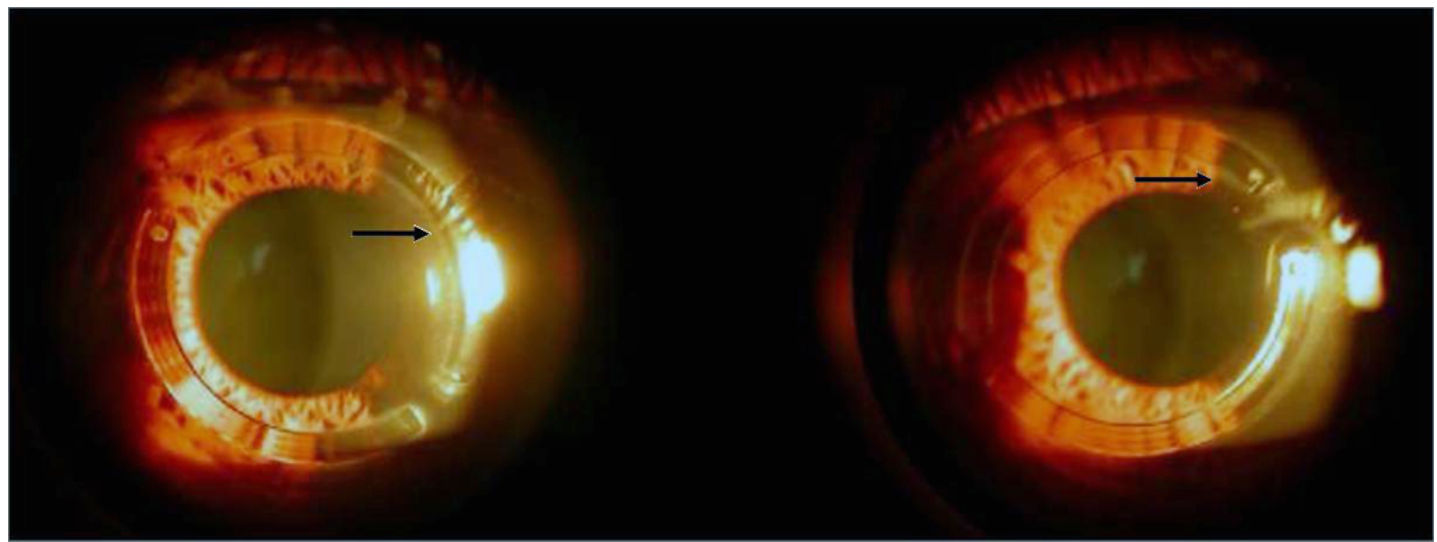

Fig. (6). Slit lamp examination nine years post-intacs implantation revealed deposits in the lamellar channel around the segments of both eyes (as shown by the arrows).

Central corneal images revealed with confocal corneal microscopy normal epithelial cells, regular subbasal and subepithelial nerve plexus, bright keratocyte's nuclei against the darker background of the acellular part in the stroma, and normal endothelial cells. Corneal images adjacent to the segments showed normal epithelial structure and subepithelial nerve plexus. When focused on the top of the stromal tunnel, excessive scattering was observed along with dark abnormal lines adjacent to the channel (Fig. 7A), while deeper images exhibited a highly reflective interface as well as oval shaped particles of various sizes and stretched keratocytes (Fig. 7B, C).

\section{DISCUSSION}

Even though, intra-stromal corneal ring segments (Intacs, Addition Technology, Inc, Fremont, California, USA) were designed at first to correct low myopia by flattening the central corneal curvature [8], they were later used as a treatment modality for keratoconus. There are many studies in the literature demonstrating pleasing results concerning the refractive outcomes post segment implanation; while there is a report on a ten-year follow up of $360^{\circ}$ intra-stromal corneal rings for myopia indicting satisfactory refractive outcomes [9], there is no documentation of a nine year follow up for the management of keratoconus.

Two years after intacs implantation, the patient had improved UCVA in both eyes. Nine years postoperatively, refractive and topographic stability was observed in comparison with two years' follow-up findings. Slit-lamp examination revealed deposits in the lamellar channel around the temporal segment of the right eye but no long-term, sight-threatening complications due to Intacs implantation. 

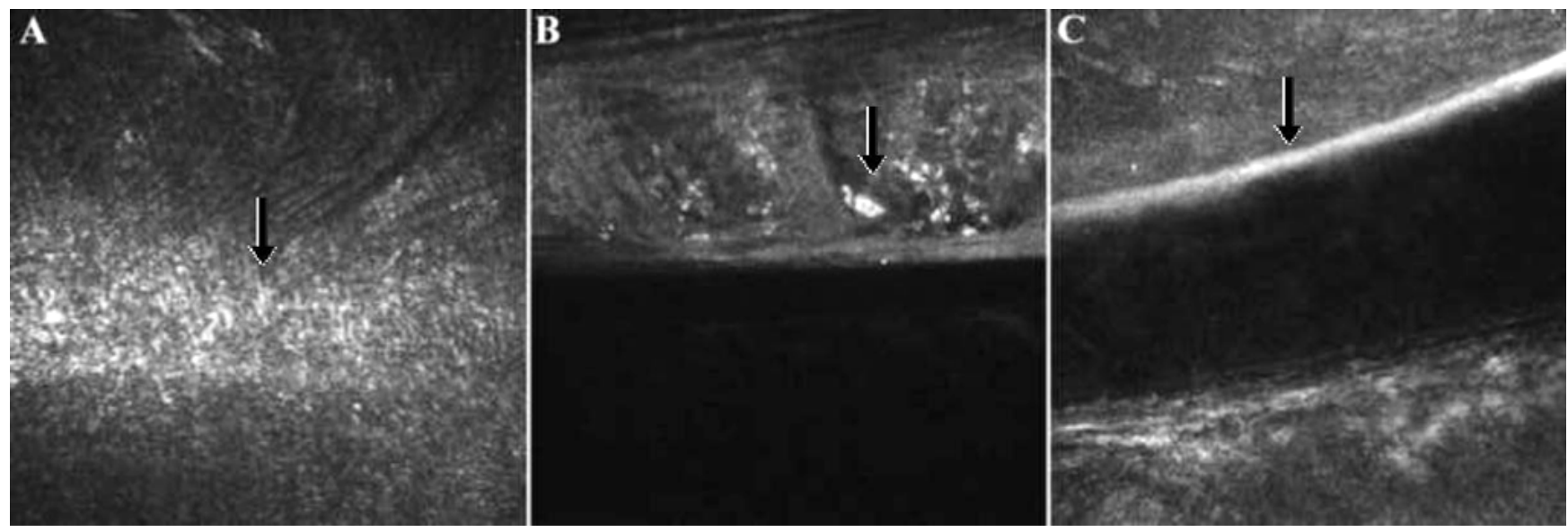

Fig. (7). Confocal microscopy images nine years post-intacs implantation: (A) Anterior channel fibrosis and increased scattering. (B) Microdeposits and abnormal lamellar structure adjacent to intac, OD. (C) Stretched keratocytes and increased scattering indicative of channel boundaries, OS (as shown by the arrows).

While the central corneal confocal images exhibited characteristics of a normal cornea [10], the increased scattering near or at the channel was probably related to fibrosis, increased cellular density, and the absence of organized lamellar structure $[11,12]$. Moreover, the deposits on or adjacent (both inward and outward) to the segments are most probably related to intracellular lipids accumulations or morphologically abnormal cellular structures $[11,12]$.

A major limitation of this case report is that the patient presented suffered from early stage keratoconus (stage 1). Keratoconus stage may affect Intacs outcomes and the refractive results that our patient achieve nine yeas post operatively, may not be extrapolated to all keratoconic patients. Another limitation is that the patient presented here underwent bilateral treatment; therefore we may not conclude that ring implantation inhibited the progression of keratoconus. Furthermore, stage 1 keratoconus may be stable in time independently to intacs implantation; therefore the stabilization of the ectatic corneal disorder in this patient may not be attributed to the intacs.

In conclusion, we present the outcome of a keratoconic patient nine years after bilateral Intacs implantation for the management of mild keratoconus. There was significant improvement and postoperative stability in patient's visual acuity without long-term, sight-threatening complications.

\section{COMPETING INTERESTS}

The authors have no financial or proprietary interest in any materials or methods described herein.

Dr. George D. Kymionis has received for the academic year 2009, a scholarship of 15000 euro from the 'Hellenic society of intraocular implants and refractive surgery' as an economic aid for a fellowship program.

\section{AUTHORS' CONTRIBUTIONS}

GDK: Manuscript idea, critical revisions to manuscript.

MAG: Collection of clinical data, manuscript preparation.

VFD: Manuscript preparation, confocal microscopy image analysis.

AIP: Confocal expertise and image analysis

IGP: Head of the department, revision of manuscript.

\section{REFERENCES}

[1] Rabinowitz YS. Keratoconus. Surv Ophthalmol 1998; 42: 297-319.

[2] Binder PS, Lindstrom RL, Stulting RD, et al. Keratoconus and corneal ectasia after LASIK. J Refract Surg 2005; 21:749-52.

[3] Tan DTH, Por Yong-Ming. Current treatment options for corneal ectasia. Curr Opin Ophthalmol 2007; 18: 284-9.

[4] Siganos CS, Kymionis GD, Kartakis N, et al. Managment of keratoconus with intacts. Am J Ophthalmol 2003; 135: 64-70.

[5] Colin J, Malet FJ. Intacs for the correction of keratoconus: twoyear follow-up. J Cataract Refract Surg 2007; 33: 69-74.

[6] Kymionis GD, Siganos CS, Tsiklis NS, et al. Long-term follow-up of intacs in keratoconus. Am J Ophthalmol 2007; 143: 236-44.

[7] Krumeich JH, Daniel J, Knulle A. Live-epikeratophakia for keratoconus. J Cataract Refract Surg 1998; 24: 456-63.

[8] Schanzlin DJ, Asbell PA, Burris TE, Durrie DS. The intrastromal corneal ring segments: Phase II results for the correction of myopia. Ophthalmology 1997; 104: 1067-78.

[9] Schwartz AP, Tinio BO, Esmail F, et al. Ten year follow up of $360^{\circ}$ intrastromal corneal rings for myopia. J Refract Surg 2006; 22: 878-83.

[10] Stave J, Zinser G, Grummer G, Guthoff R. Modified Heidelberg Retina Tomograph HRT. Initial results of in vivo presentation of corneal structures. Ophthalmology 2002; 99: 276-80.

[11] Twa MD, Ruckhofer J, Kash RL, Costello M, Schanzlin DJ. Histological evaluation of corneal stroma in rabbits after intrastromal corneal ring implantation. Cornea 2003; 22: 146-52.

[12] Twa MD, Kash RL, Costello M, Schanzlin DJ. Morphological characteristics of lamellar channel deposits in the human eye: a case report. Cornea 2004; 23: 412-20. 